\title{
Continuum Extrapolation of Moments of Nucleon Quark Distributions in Full QCD
}

LHPC and SESAM Collaborations:

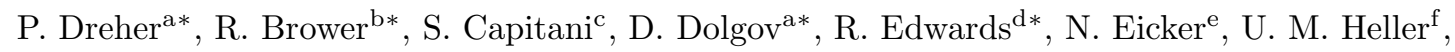
Th. Lippert ${ }^{\mathrm{e}}$, J. W. Negele ${ }^{\mathrm{a} *}$, A. Pochinsky ${ }^{\mathrm{a} *}$, D. B. Renner ${ }^{\mathrm{a}}$, K. Schilling ${ }^{\mathrm{e}}$

${ }^{a}$ Center for Theoretical Physics, Massachusetts Institute of Technology, Cambridge, MA 02139, USA

${ }^{\mathrm{b}}$ Department of Physics, Boston University, Boston, MA 02215, USA

${ }^{\mathrm{c} J o h n}$ von Neumann Institut für Computing, DESY, D-15738 Zeuthen, Germany

${ }^{d}$ Thomas Jefferson National Accelerator Facility, Newport News, VA 23606, USA

${ }^{e}$ Department of Physics, University of Wuppertal, D-42097 Wuppertal, Germany

${ }^{\mathrm{f}}$ CSIT, Florida State University, Tallahassee, FL 32306-4120, USA

Moments of light cone quark density, helicity, and transversity distributions are calculated in unquenched lattice QCD at $\beta=5.5$ and $\beta=5.3$ using Wilson fermions on $16^{3} \times 32$ lattices. These results are combined with earlier calculations at $\beta=5.6$ using SESAM configurations to study the continuum limit.

\section{INTRODUCTION}

Calculation of moments of light cone parton distributions using lattice QCD provides the only known way to calculate the quark and gluon structure of the nucleon from first principles. Quenched calculations of flavor non-singlet moments of parton distributions yield well-known discrepancies with experiment [1], so serious effort has been undertaken to calculate these moments in full QCD [2,3]. To augment the calculations in ref. [3] at $\beta=5.6$, this work reports calculations at $\beta=5.5$ and $\beta=5.3$ to study the approach to the continuum limit using Wilson fermions.

Moments of the quark density, helicity, and transversity distributions are related to the following matrix elements of twist-2 operators:

$$
\begin{aligned}
& \left\langle x^{n-1}\right\rangle_{q_{r}} P_{\mu_{1}} \cdots P_{\mu_{n}} \\
& \quad=\frac{1}{4}\left\langle P S\left|\left(\frac{i}{2}\right)^{n-1} \bar{\psi} \gamma_{\left\{\mu_{1}\right.} \stackrel{\leftrightarrow}{D}_{\mu_{2}} \cdots \stackrel{\leftrightarrow}{D}_{\left.\mu_{n}\right\}} \psi\right| P S\right\rangle
\end{aligned}
$$

\footnotetext{
* Support provided through the U.S. Department of Energy Cooperative Agreement DE-FC02-94ER40818 and contracts DE-FG02-91ER40676, DE-FG02-97ER41022, and DE-AC05-84ER40150
}

$$
\begin{aligned}
& \left\langle x^{n}\right\rangle_{\Delta q_{r}} S_{\{\sigma} P_{\mu_{1}} \cdots P_{\left.\mu_{n}\right\}} \\
& \quad=-\frac{n+1}{2}\left\langle P S\left|\left(\frac{i}{2}\right)^{n} \bar{\psi} \gamma_{5} \gamma_{\{\sigma} \stackrel{\leftrightarrow}{D}_{\mu_{1}} \cdots \stackrel{\leftrightarrow}{D}_{\left.\mu_{n}\right\}} \psi\right| P S\right\rangle \\
& \left\langle x^{n}\right\rangle_{\delta q_{r}} S_{[\mu} P_{\{\nu]} P_{\mu_{1}} \cdots P_{\left.\mu_{n}\right\}} \\
& \quad=\frac{m_{N}}{2}\left\langle P S\left|\left(\frac{i}{2}\right)^{n} \bar{\psi} \gamma_{5} \sigma_{\mu\{\nu} \stackrel{\leftrightarrow}{D}_{\mu_{1}} \cdots \stackrel{\leftrightarrow}{D}_{\left.\mu_{n}\right\}} \psi\right| P S\right\rangle
\end{aligned}
$$

We calculate four moments calculable using zero-momentum nucleon states: the spin averaged momentum fraction $\langle x\rangle_{u-d}$, the axial charge $\langle 1\rangle_{\Delta u-\Delta d}$, the longitudinal spin momentum fraction $\langle x\rangle_{\Delta u-\Delta d}$, and the tensor charge $\langle 1\rangle_{\delta u-\delta d}$.

\section{METHODOLOGY}

To facilitate direct comparison, we follow the same methodology as in ref. [3], which used SESAM configurations at $\beta=5.6$ on a $16^{3} \times 32$ lattice with unimproved Wilson fermions. In particular, we calculated the ratios of three-point to two point functions using sequential Wuppertal smeared sources with the nucleon projected to momentum zero and Dirichlet boundary conditions in the time direction. Renormalization constants to convert from lattice regularization to the 
Table 1

Moments of the quark distributions

\begin{tabular}{cccccc}
\hline$\beta$ & $\kappa$ & $\langle x\rangle_{u-d}$ & $\langle 1\rangle_{\Delta u-\Delta d}$ & $\langle x\rangle_{\Delta u-\Delta d}$ & $\langle 1\rangle_{\delta u-\delta d}$ \\
\hline 5.3 & .1665 & $.232(6)$ & $1.202(25)$ & $.257(9)$ & $1.353(21)$ \\
5.3 & .1670 & $.253(7)$ & $1.225(26)$ & $.276(10)$ & $1.312(23)$ \\
\hline 5.5 & .1592 & $.230(3)$ & $1.180(12)$ & $.262(4)$ & $1.273(9)$ \\
5.5 & .1596 & $.238(5)$ & $1.140(17)$ & $.265(5)$ & $1.277(13)$ \\
\hline 5.6 & .1560 & $.247(9)$ & $1.190(16)$ & $.297(6)$ & $1.325(13)$ \\
5.6 & .1565 & $.242(9)$ & $1.223(20)$ & $.298(7)$ & $1.287(18)$ \\
5.6 & .1570 & $.263(13)$ & $1.094(25)$ & $.283(9)$ & $1.261(26)$ \\
\hline
\end{tabular}

$\overline{M S}$ scheme at $4 \mathrm{GeV}^{2}$ were calculated perturbatively.

At $\beta=5.6$ the smearing was optimized to maximize the overlap of the source with the nucleon ground state, yielding $N=50$ smearing steps, and the source-sink separation was chosen to be 12 lattice steps to minimize the statistical errors subject to the requirement of a well-defined plateau in the three-point function.

At $\beta=5.5$ to keep the physical source size and the source-sink distance constant, we used 30 smearing steps and a separation of 10 lattice sites. For $\kappa=0.1592$ we used 413 hybrid Monte Carlo configurations, each separated by 20 trajectories, and at $\kappa=0.1596$, we used 243 configurations separated by 10 trajectories.

At $\beta=5.3$ we used 25 smearing steps and a source-sink separation of 9 lattice sites. At $\kappa=$ 0.1665 , we used 225 configurations separated by 15 trajectories, and at $\kappa=0.1670$ we used 240 configurations separated by 10 trajectories.

\section{CHIRAL EXTRAPOLATION}

Since the pion cloud contributes significantly to many of the operators characterizing form factors and moments of structure functions, it is reasonable to expect large, qualitative differences between the behavior of matrix elements in the heavy quark regime where current lattice calculations are carried out and in the light quark regime pertinent to physical pions. A physical description of the transition between these two regimes is given by the following interpolation formula [3, [4], which incorporates the leading nonanalytic behavior in the pion mass specified by chiral perturbation theory:

$$
\begin{aligned}
& \left\langle x^{n}\right\rangle_{u-d} \\
& \quad=a_{n}\left[1-\frac{\left(3 g_{A}^{2}+1\right) m_{\pi}^{2}}{\left(4 \pi f_{\pi}\right)^{2}} \ln \left(\frac{m_{\pi}^{2}}{m_{\pi}^{2}+\mu^{2}}\right)\right]+b_{n} m_{\pi}^{2} .
\end{aligned}
$$

The parameter $\mu$ specifies the physical scale of the three quark core that serves as the source term for the pion cloud, and a single value of this parameter of the order of $500 \mathrm{MeV}$ resolves the discrepancy in the lowest three moments $\langle x\rangle_{u-d}$, $\left\langle x^{2}\right\rangle_{u-d}$, and $\left\langle x^{3}\right\rangle_{u-d}$ as well as the nucleon magnetic moment. The best fit [3] to the $\beta=5.6$ data for $\langle x\rangle_{u-d}$ is shown by the solid line in the upper panel of Figure 1. Of particular importance for the present work is the fact that this interpolation formula is nearly constant in the regime in which all our lattice calculations are carried out. Hence, even though we only have data at two quark masses for $\beta=5.5$ and for $\beta=5.3$ we may study the approach to the continuum limit by determining the best least squares fit of the parameter $a_{1}$ at each $\beta$. Analogous interpolation formulae for $\langle 1\rangle_{\Delta u-\Delta d},\langle x\rangle_{\Delta u-\Delta d}$, and $\langle 1\rangle_{\delta u-\delta d}$ are presented in ref. [5] and used to fit the data in the lower three panels of Figure 1. Note that, in contrast to the spin averaged momentum fraction, the three spin-dependent moments are far less sensitive to the pion mass because of strong cancellation between the the nucleon and delta contributions in chiral perturbation theory.

\section{RESULTS}

The data are presented in Table 1. The results of least squares fits of the interpolation formulae to the data at $\beta=5.6,5.5$, and 5.3 are shown in Figure 1 by the solid, dashed, and dotted lines respectively. For each of the four operators, we see that the fractional shift in these lines is small as one changes $\beta$. Especially since the lattice spacing is quite large at $\beta=5.3$, these results show that the $\mathcal{O}(a)$ corrections for unimproved Wilson fermions are small and benign for the operators considered in this work. This provides solid evidence that the extrapolation to the continuum limit is reasonably close to the results at $\beta=5.6$. By ruling out significant $a$ dependence, this re- 


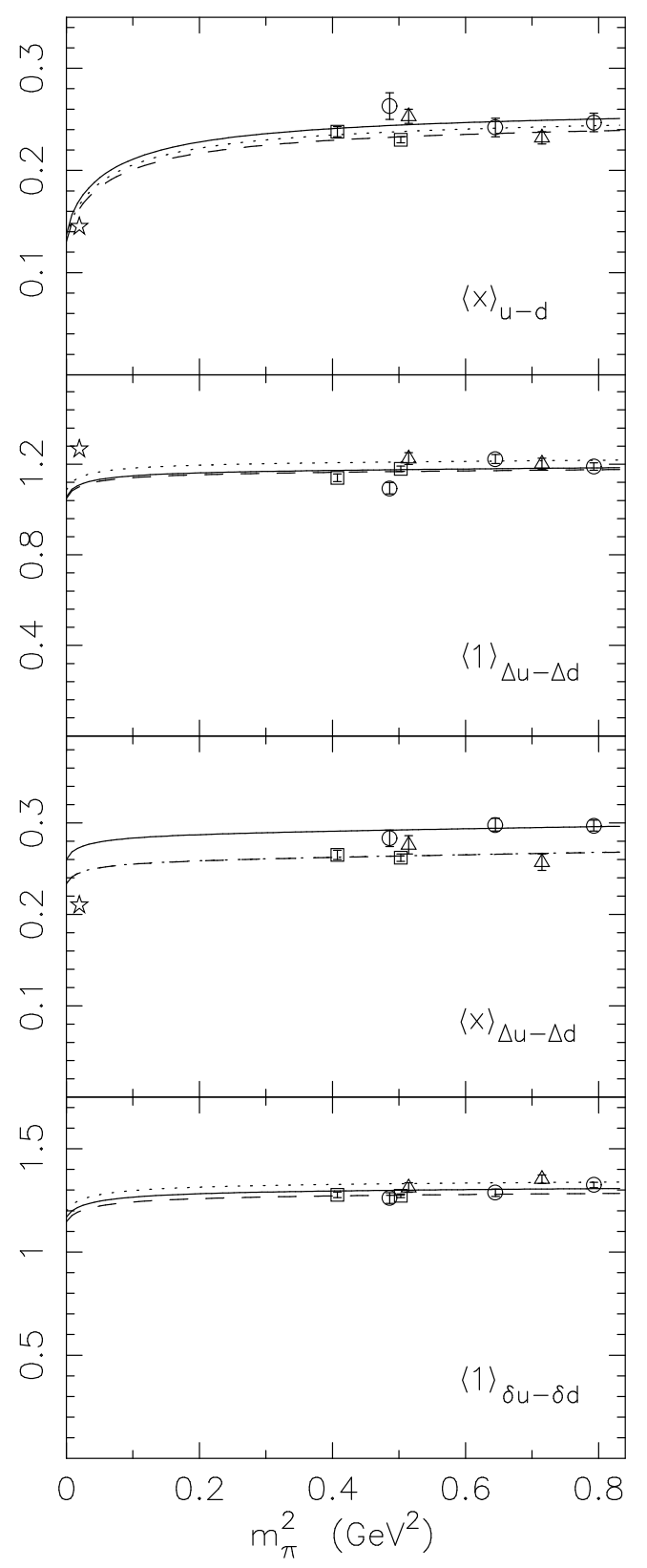

Figure 1. Moments of quark distributions and interpolation formula fits at $\beta=5.6$ (circles and solid lines), 5.5 (squares and dashed lines), and 5.3 (triangles and dotted lines). sult thus further substantiates the physical argument that the origin of the discrepancy between phenomenology and naive linear extrapolation of lattice moments of parton distributions must lie in the physics of the chiral extrapolation.

\section{ACKNOWLEDGEMENT}

These calculations utilized some configurations at $\beta=5.3$ from the HEMCGC collaboration and at $\beta=5.5$ from the SCRI group. Additional production of hybrid Monte Carlo configurations and measurements of operators were performed on the MIT alpha cluster, the JLab alpha cluster, and the JLab QCDSP computer.

\section{REFERENCES}

1. M. Göckeler, R. Horsley, E.M. Ilgenfritz, H. Perlt, P. Rakow, G. Schierholz, A. Schiller, hep-lat/9508004; Göckeler et. al, hep-lat/708270, 9711245, 9909253, and 0011091.

2. R. Horsley, Plenary talk this conference, (2002).

3. D. Dolgov, R. Brower, S. Capitani, P. Dreher, R.G. Edwards, N. Eicker, U.M. Heller, Th. Lippert, A. Pochinsky, D.B. Renner, K. Schilling, Phys. Rev. D 66, 034506 (2002) hep-lat/0201021.

4. W. Detmold, W. Melnitchouk, J.W. Negele, D.B. Renner, and W.W.Thomas, Phys. Rev. Lett. 87, 172001 (2001) hep-lat/0103006.

5. W. Detmold, W. Melnitchouk, A.W. Thomas, hep-lat/0206001 (2002). 